\title{
A xylose-utilizing Saccharomyces cerevisiae strain serves as a better host for producing acetyl-CoA derived n-butanol
}

\author{
Yeon Jung Lee ${ }^{1}$, Phuong Hoang Nguyen Tran ${ }^{1}$, Ja Kyong Ko ${ }^{1}$, Gyeongtaek Gong ${ }^{1}$, \\ youngsoon $\mathrm{um}^{2}$, Sung Ok Han ${ }^{3}$, and Sun-Mi Lee ${ }^{1}$ \\ ${ }^{1}$ Korea Institute of Science and Technology \\ ${ }^{2}$ Korea Institute of Science and Engineering \\ ${ }^{3}$ Korea University
}

September 25, 2021

\begin{abstract}
Efficient xylose catabolism in engineered Saccharomyces cerevisiae enables more economical lignocellulosic biorefinery with improved production yields per unit of biomass. Yet, the product profile of glucose/xylose co-fermenting S. cerevisiae is mainly limited to bioethanol and a few other chemicals. Here, we introduced an n-butanol-biosynthesis pathway into a glucose/xylose co-fermenting S. cerevisiae strain (XUSEA) to evaluate its potential on the production of acetyl-CoA derived products. Higher n-butanol production of glucose/xylose co-fermenting strain was explained by the transcriptomic landscape, which revealed strongly increased acetyl-CoA and NADPH pools when compared to a glucose fermenting wild-type strain. The acetate supplementation expected to support acetyl-CoA pool further increased n-butanol production, which was also validated during the fermentation of lignocellulosic hydrolysates containing acetate. Our findings imply the feasibility of lignocellulosic biorefinery for producing fuels and chemicals derived from a key intermediate of acetyl-CoA through glucose/xylose co-fermentation.
\end{abstract}

\section{Introduction}

Lignocellulosic biomass offers a sustainable and environmentally friendly source of raw materials for producing fuels and chemicals (Ko and Lee, 2018). Commercial bioethanol production has been achieved using the yeast Saccharomyces cerevisiae and sugar- or starch-based biomass. To improve the economic feasibility of lignocellulosic biorefinery, S. cerevisiae strains have been engineered to co-ferment glucose and xylose, the most abundant hexose and pentose sugars in lignocellulosic hydrolysates, respectively, resulting in significantly improved lignocellulosic bioethanol yields, titers, and productivity (Hoang Nguyen Tran et al., 2020). In addition, increasing efforts have also been devoted to expanding the product profile of $S$. cerevisiae to include advanced fuels and chemicals demonstrating the great potential of microbial cell factories for biorefinery (Ekas et al., 2019; Kim et al., 2020). Yet, the production of non-native products using both glucose and xylose derived from lignocellulosic hydrolysates has been less explored. Thus, it is necessary to evaluate the feasibility of sustainable-biorefinery concepts based on lignocellulosic biomass, which is the most abundant and sustainable resource.

Efficient glucose/xylose co-fermentation enables complete utilization of all available sugars in lignocellulosic biomass, which increases the overall conversion yield in lignocellulosic biorefinery. By incorporating xylose into the substrate used by S. cerevisiae, the overall conversion yield could be increased by up to $84 \%$ during lignocellulosic bioethanol production (Ko et al., 2018). In addition, xylose metabolism supported the diversion of metabolic flux toward non-ethanol products in an ethanol-producing $S$. cerevisiae yeast strain (Li et al., 2019). Recently, xylose-utilizing strains have been suggested as promising microbial cell factories for producing acetyl-CoA-derived products (Kwak and Jin, 2017), due to their altered cellular metabolism. 
Therefore, it is of interest to confirm the advantages of glucose/xylose co-fermentation in producing fuels and chemicals derived from acetyl-CoA at the phenotypic and transcriptional levels.

As a core intermediate in central carbon metabolism, acetyl-CoA can be converted into various fuels and chemicals. Yeast strains have been suggested to be better hosts for producing acetyl-CoA-derived products than other microbial hosts (Sun and Alper, 2020). With limited metabolic flux through cytosolic acetyl-CoA, however, the successful production of fuels and chemicals derived from acetyl-CoA has been rarely demonstrated in S. cerevisiae. Specifically, the production of acetyl-CoA-derived n-butanol, a short chain alcohol similar to ethanol, has been limited to less than $1 \mathrm{~g} / \mathrm{L}$, even with higher butanol tolerance of $S$. cerevisiae when compared to other non-native bacterial hosts (González-Ramos et al., 2013; Hong and Nielsen, 2012; Knoshaug and Zhang, 2009). Engineering S. cerevisiae for n-butanol production has been based mainly on the reverse- $\beta$-oxidation pathway ofClostridium sp., which naturally produce butanol. Therefore, successful n-butanol production would also enable successful incorporation of the reverse- $\beta$-oxidation pathway, an economic route for medium-chain fatty acids and alcohols with numerous industrial applications, into the cellular network of $S$. cerevisiae.

Here, we sought to evaluate the potential of a glucose/xylose co-fermenting strain of S. cerevisiae as a production host for acetyl-CoA-derived n-butanol in lignocellulosic biorefinery concept. To this end, an n-butanol-biosynthesis pathway was introduced in XUSEA, a previously developed $S$. cerevisiae strain that simultaneously ferments glucose and xylose (Tran Nguyen Hoang et al., 2018; Hoang Nguyen Tran et al., 2020). The transcription profile of the XUSEA strain was compared to that of the wild-type strain to understand the modified cellular network caused by the introduction of a heterologous route for bioconverting xylose into butanol. Butanol production in the glucose/xylose co-fermenting strain was further improved by supplementation with acetic acid, a major by-product generated during lignocellulosic biomass-pretreatment process. Finally, we successfully demonstrated lignocellulosic n-butanol production by the glucose/xylose cofermenting strain, XUSAEA-B. Consequently, the results of this study show the potential of a glucose/xylose co-fermenting strain in the production of acetyl-CoA-derived n-butanol from lignocellulosic biomass.

\section{Materials and Methods}

\subsection{Strains and culture conditions}

All strains used in this study are shown in Table 2. The yeast strains used in this study were isogenic with respect to $S$. cerevisiaeS288C BY4741. The yeast strains were routinely cultivated at $30^{\circ} \mathrm{C}$ in yeast synthetic complete (YSC) medium composed of xylose (and/or glucose), $6.7 \mathrm{~g} / \mathrm{L}$ of yeast nitrogen base (Difco, Detroit, MI, USA), and CSM-HIS-LEU-URA (MP Biomedicals, Solon, Ohio, USA). Escherichia coli DH10 $\beta$ cells were employed for DNA manipulation and were cultured at $37^{\circ} \mathrm{C}$ in LB medium supplemented with $100 \mu \mathrm{g} / \mathrm{mL}$ ampicillin.

\subsection{Plasmid construction}

All plasmids used in this study are shown in Table 3. The homologous genes used in this study were ERG10 and ETR1, and the heterologous genes were EutE from E. coli , $K r$ and $H t d$ from Yarrowia lipolytica , and $H b d, C r t, A d h E 2$, and BdhB from Clostridium acetobutylicum. The start codon of BdhB was changed from GTG to ATG, and the mitochondria-targeting sequences of the Kr, Htd, and Etr1 proteins were predicted and excluded using TargetP software version 1.1 (http://www.cbs.dtu.dk/services/TargetP/) and the MITOPROT online tools (https://ihg.gsf.de/ihg/mitoprot.html) according to the reference by Lian and Zhao (2015). Each gene cassette was cloned into an expression vector using the Gibson Assembly method and introduced in S. cerevisiae using the Frozen-EZ Yeast Transformation II Kit (Zymo Research), according to the manufacturer's instructions.

\subsection{Predicting mitochondria-targeting sequences}

The online MITOPROT tool was used to predict and exclude mitochondria-targeting signal peptide sequences, which were further validated using TargetP, version 1.1 (Lian and Zhao, 2015). The 
nucleotide sequences encoding the excluded signal peptides are as follows: TTCCGACTCACCACTGCCCGAATTGCTTCTGTGCGAGGCTTCTCCACCTCCGCCAGCCTGTCC (for $\mathrm{Kr}$ ), CGAAGCCTATATATAAACGTTCCGGGTCTTTTTCCTTCCACCTCTCTAGCACGAGAA (for Htd ), and CTTCCCACATTCAAACGTTACATG (for Etr1 ). Additionally, an intron sequence of $H t d$ was excluded along with mitochondria-targeting sequence.

2.4. Fermentation For seed cultures, yeast cells from a glycerol stock were inoculated in YSC medium containing $2 \%$ glucose. The yeast cells were then transferred to fresh YSC medium containing $2 \%$ glucose, $2 \%$ xylose, or $2 \%$ glucose plus $2 \%$ xylose with an inoculum size of $5 \%$, and grown aerobically in flasks for 1.5-2 days. Subsequently, the yeast cells were harvested and finally transferred to fresh YSC medium. The $\mathrm{pH}$ of the fermentation medium was maintained by adding $70 \mathrm{mM}$ phthalate buffer ( $\mathrm{pH} 5.0$ ) or $80 \mathrm{mM}$ phosphate buffer ( $\mathrm{pH}$ 6.5). Micro-aerobic fermentation was carried out in 125-mL serum bottles with a final working volume of $40 \mathrm{~mL}$ at a low cell density (initial optical density [OD] of 0.2) and a high cell density (initial OD of 15). The serum bottles were capped with rubber stoppers, with a needle for carbon dioxide release during fermentation. Lignocellulosic biomass hydrolysates (Miscanthus sacchariflorus Goedae-Uksae 1 ), pretreated with dilute acid, were purchased from Sugaren Co., Ltd. (Korea).

\subsection{Analytical methods}

Cell growth was analyzed by measuring the OD at $600 \mathrm{~nm}$ with a Cary 60 Bio UV-Vis spectrometer (Agilent Technologies, USA). Glucose and xylose concentrations were analyzed using a high-performance liquid chromatography system (HPLC 1260 Infinity, Agilent Technologies, CA, USA) equipped with a refractive index detector, using a Hi-Plex H column (Agilent Technologies, Palo Alto, CA, USA). The system was operated using $5 \mathrm{mM} \mathrm{H}_{2} \mathrm{SO}_{4}$ as the mobile phase at a flow rate of $0.6 \mathrm{~mL} / \mathrm{min}$, and the column temperature was maintained at $65^{\circ} \mathrm{C}$. Ethanol, n-butanol, and acetate concentrations were analyzed using a gas chromatograph instrument (Agilent Technologies, CA, USA) equipped with a flame ionization detector, using an HP-INNOWax polyethylene glycol column $(30 \mathrm{~m} \times 0.32 \mu \mathrm{m} \times 0.25 \mu \mathrm{m})$.

\section{Results}

\subsection{Introduction of a n-butanol production pathway in a glucose/xylose co-fermenting strain}

To develop an S. cerevisiae strain capable of efficient n-butanol production, genes that were previously reported to support butanol biosynthesis were introduced in an efficient glucose/xylose co-fermenting strain, XUSEA, with various combinations (Hoang Nguyen Tran et al., 2020). Specifically, $H b d$ and $C r t$ originated from C. acetobutylicum (a representative n-butanol-producing bacterium), and the orthologous genes ( $K r$ and $H t d$, respectively) originated from $Y$. lipolytica, a yeast with an efficient $\beta$-oxidation pathway. These genes

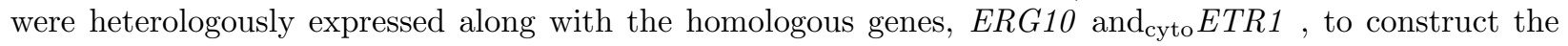
reverse- $\beta$-oxidation pathway, thereby enabling the conversion of acetyl-CoA into butyryl-CoA (Dellomonaco et al., 2011; Lian and Zhao, 2015). To generate a complete butanol production pathway, Adhe2 or BdhB from C. acetobutylicum were heterologously expressed with or withoutEutE from E. coli (Fig. 1a). Heterologous expression of these C. acetobutylicum genes increased butanol production more than heterologous expression of the indicated $Y$. lipolyticagenes, even without codon optimization. When expressing heterologous genes, it is often expected that genes originating from yeasts or other eukaryotic cells will be expressed at higher levels in eukaryotic cells (such as S. cerevisiae). However, the distance in a phylogenetic tree does not seem to guarantee better performance, when expressed in a heterologous host.

The choice of the alcohol dehydrogenase used, either Adhe2 or BdhB, did not significantly affect the butanol titer. Although AdhE2 is a bi-functional enzyme that converts butyryl-CoA into butyraldehyde and then to n-butanol, it cannot fully support butanol production withoutEutE expression, suggesting that sufficient enzyme levels are required for each step in n-butanol synthesis. Of the heterologous gene combinations tested in glucose/xylose co-fermenting XUSEA strain, the set comprised of $H d b, C r t, E u t E$, and AdhE2resulted in the highest butanol titer of $7.2 \mathrm{mg} / \mathrm{L}$, followed by the set comprised of $H d b, C r t$, EutE , and $B d h B(6.8$ $\mathrm{mg} / \mathrm{L}$ ) (Fig. 1b). Based on the similar butanol titer with a half size of $B d h B$ compared to Adhe2, the set consisting of $E R G 10, H b d, C r t$, cyto $E T R 1, B d h B$, and EutE was selected as the combination of choice for 
constructing an n-butanol production pathway for further experiments.

The butanol fermentation performances of a xylose-utilizing strain (XUSEA-B) was compared to that of the wild-type strain (WT-B), both of which expressed the selected butanol production pathway genes during glucose fermentation, xylose fermentation, and glucose/xylose co-fermentation (Fig. 2). During glucose fermentation, the XUSEA-B strain showed 2.1-fold higher n-butanol production than the WT-B strain (14.2 $\mathrm{mg} / \mathrm{L}$ and $6.6 \mathrm{mg} / \mathrm{L}$, respectively; Fig. 2a). The XUSEA-B strain produced the same amount of n-butanol during xylose fermentation, whereas the WT-B strain produced no n-butanol because it could not utilize xylose. During glucose/xylose co-fermentation, XUSEA-B produced $26.3 \mathrm{mg} / \mathrm{L}$ of n-butanol within $72 \mathrm{~h}$, which was 3.9-fold higher than that produced by WT-B $(6.7 \mathrm{mg} / \mathrm{L}$; Fig. 2a), suggesting that the xyloseutilizing strain served as a better host for producing n-butanol, an acetyl-CoA-derived product.

\subsection{Transcriptomic landscape revealed the redirected carbon flux for improved butanol pro- duction in a xylose-utilizing strain.}

To understand the mechanism underlying efficient n-butanol production by the xylose-utilizing strain, we analyzed the global transcript profiles of XUSEA-B and WT-B during glucose and glucose/xylose fermentation. Compared with WT-B, the XUSEA-B strain showed marked differences in the gene-expression landscape (170 upregulated and 84 downregulated genes with glucose fermentation versus 172 upregulated and 82 downregulated genes with mixed-sugar fermentation) (Fig. 3). Transcriptional changes in genes associated with core carbon metabolism and linked with the butanol production pathway were similar between both strains, despite the use of different carbon sources. The XUSEA-B strain appeared to show elevated central carbon flux through the n-butanol production pathway, resulting from pathway upregulation due to increased cofactor generation and reduced use of the acetyl-CoA pool. The ZWF1,SOL3, and GND1 genes (involved in the oxidative pentose phosphate pathway, from which the factor NADPH is generated), were upregulated in XUSEA-B compared to WT-B by 2.81-, 4.11-, and 7.82-fold during glucose fermentation and by 3.74-, 5.54-, and 9.93-fold during glucose/xylose co-fermentation, respectively (Fig. 3, Table 1).

It should be noted that genes involved in the non-oxidative pentose phosphate pathway (such as RPE1 and TAL1 ) and XKS1 were highly upregulated in the XUSEA-B strain, compared to the wild-type strain (Fig. 3, Table 1). These differences were mainly due to the genetic background of XUSEA, in which the above-mentioned genes were overexpressed (Hoang Nguyen Tran et al., 2020; Tran Nguyen Hoang et al., 2018). However, it is noteworthy that RPE1 mRNA expression was upregulated by approximately 100 -fold in the XUSEA-B strain. This finding suggests that carbon flux was enhanced through glycerate-3phosphate, an interconnection point between the oxidative and non-oxidative pentose phosphate pathways. The transcription of $P D C 6$, which is involved in converting pyruvate to acetaldehyde, was also strongly upregulated suggesting that the acetyl-CoA pool was increased in XUSEA-B. In contrast, the FBA1, TDH2 , andENO2 genes (involved in glycolysis), were downregulated more than 2-fold in XUSEA-B, leading to a low carbon flux through central carbon metabolism via glycolysis.

\subsection{Supplementation with acetate improved n-butanol production by $S$. cerevisiae}

After confirming the positive effect of the increased acetyl-CoA pool in XUSEA-B, based on transcriptomic analysis, the effect of acetate supplementation on n-butanol production was investigated as a strategy for supplying additional acetyl-CoA. To this end, n-butanol production by XUSEA-B was evaluated during glucose and/or xylose fermentation supplemented with various concentrations of acetate $(0 \mathrm{~g} / \mathrm{L}, 1 \mathrm{~g} / \mathrm{L}$, or 2 $\mathrm{g} / \mathrm{L})$. During glucose fermentation, acetate supplementation increased n-butanol production by XUSEA-B (Fig. 4). XUSEA-B strains produced $40 \%$ or $28 \%$ more n-butanol in the presence of 1 or $2 \mathrm{~g} / \mathrm{L}$ acetate, respectively. In contrast, acetate supplementation did not result in increased n-butanol production during xylose fermentation. The production of n-butanol decreased by $56 \%$ in the presence of $1 \mathrm{~g} / \mathrm{L}$ acetate during $48 \mathrm{~h}$ of xylose fermentation. No n-butanol was produced in the presence of $2 \mathrm{~g} / \mathrm{L}$ acetate in the medium because the cell growth was significantly inhibited (data not shown). Interestingly, the negative effect of acetate supplementation on n-butanol production during xylose fermentation was diminished in the presence of glucose. During glucose/xylose co-fermentation, n-butanol production was reduced by $23 \%$ in the presence 
of $1 \mathrm{~g} / \mathrm{L}$ acetate (Fig. $4 \mathrm{~b})$.

Given the both positive and negative effects of acetate supplementation on n-butanol production, we introduced the n-butanol production pathway into an acetate-tolerant glucose/xylose co-fermenting strain of XUSAE (Ko et al., 2019), thus generating XUSAE-B. To promote the conversion of acetate into acetyl-CoA, a heterologous acetate-utilization pathway was additionally integrated into XUSAE-B by overexpressing a mutant version of $\mathrm{ACS}^{L 641 P}$ from Salmonella enterica and AADH from E. coli, thereby generating XUSAEA-B. The acetate-tolerant strain harboring the acetate-utilization pathway showed slightly increased n-butanol production with acetate supplementation $(1 \mathrm{~g} / \mathrm{L})$ during glucose/xylose fermentation, at $\mathrm{pH} 5.0$ (Fig. 5). When the $\mathrm{pH}$ of the fermentation broth was increased from 5.0 to 6.5 , under which condition the inhibitory effect of acetate has been shown to decrease, the XUSAEA-B strain showed dramatically improved n-butanol production resulting in an n-butanol titer of $46.5 \mathrm{mg} / \mathrm{L}$.

\subsection{Successful n-butanol production was achieved from lignocellulosic hydrolysates}

Encouraged by the increased n-butanol production during glucose/xylose co-fermentation in the presence of acetate, we evaluated the n-butanol production performance of XUSAEA-B from lignocellulosic hydrolysates of Micanthus sacchariflorus Goedae-Uksae, which were prepared through a $\mathrm{H}_{2} \mathrm{SO}_{4}$-catalyzed hydrothermal process (Fig. 6). During a 21-h fermentation, XUSAEA-B completely utilized glucose and xylose, and produced $60.1 \mathrm{mg} / \mathrm{L}$ of n-butanol (Fig. 6a, c). Interestingly, n-butanol production from lignocellulosic hydrolysates was $14 \%$ higher than that from synthetic medium with same glucose/xylose concentration, but without acetate supplementation $(60.1 \mathrm{mg} / \mathrm{L}$ and $52.8 \mathrm{mg} / \mathrm{L}$ for lignocellulosic hydrolysates and synthetic medium, respectively) (Fig. 6c). To our knowledge, this study demonstrates improved butanol production from lignocellulosic hydrolysate for the first time whereas other previous studies mainly showed butanol production from synthetic media (Lian et al., 2014; Sakuragi et al., 2015; Schadeweg and Boles, 2016a, b; Swidah et al., 2015, 2018).

\section{Discussion}

Yeast engineering for lignocellulosic biorefinery has primarily focused on either the expansion of substrate ranges or product profiles through individual approaches. However, the product profiles of S. cerevisiae capable of xylose utilization are limited to only a few compounds, including isobutanol, carotene, and 1hexadecanol (Brat and Boles, 2013; Guo et al., 2016; Sun et al., 2019). Recent reports highlighting the prospect of engineering a xylose-utilizing strain as a promising host for the production of acetyl-coA derived products (Kwak and Jin, 2017) offer easier diversion of product profiles from ethanol to other products, for a glucose/xylose co-utilizing S. cerevisiae .

In this study, we demonstrated the expansion of product profiles of a glucose/xylose co-fermenting $S$. cerevisiae to include the acetyl-coA derived product, n-butanol, which is a short chain alcohol similar to ethanol, however, has only been produced by $S$. cerevisiae from glucose with limited success. Despite the numerous attempts to develop n-butanol producing $S$. cerevisiae, the production titer remained below $1 \mathrm{~g} / \mathrm{L}$ even with extensive metabolic engineering. There are several benefits associated with using a glucose/xylose cofermenting strain for n-butanol production. For instance, it has a higher metabolic flux through acetyl-CoA compared to strains capable of only utilizing glucose (Kwak and Jin, 2017; Li et al., 2019). Additionally, it has an associated higher conversion yield per unit of biomass.

These benefits were demonstrated in the current study during lignocellulosic fermentation using Miscanthus hydrolysates. Specifically, the glucose/xylose co-fermenting S. cerevisiaestrain, XUSEA-B, showed three-fold higher n-butanol content compared to the wild-type strain capable of only utilizing glucose. Given that the amount of xylose present was nearly half that of glucose, the additional n-butanol produced by XUSEA-B demonstrates a synergistic effect with an additional carbon source (xylose) in hydrolysates and an increased acetyl-CoA pool, supported by xylose catabolism in XUSEA-B.

In the study, we pointed out the increased acetyl-CoA pool of a glucose/xylose co-fermenting strain through analysis of the transcriptomic landscape in XUSEA-B, in which the increased metabolic flux was expected 
toward pyruvate, acetaldehyde, acetate, and cytosolic acetyl-CoA by re-directing the carbon flux through competing pathways such as the tricarboxylic acid (TCA) cycle, glyoxylate cycle, amino acid biosynthesis, and ethanol production (Fig 3, Tab 1). The metabolic flux through pyruvate, specifically, could be directed toward acetaldehyde based on the highly upregulated expression of PDC6 accompanied by downregulation of genes associated with pyruvate carboxylation, and those involved in the TCA cycle, glyoxylate cycle and amino acid biosynthesis employing pyruvate as a precursor. Moreover, the fate of acetaldehyde through the ethanol-fermentation pathway also appeared to be redirected toward acetate formation, based on the decreased expression of alcohol dehydrogenases encoded by $\mathrm{ADH}_{4}$ and $\mathrm{ADH}$ 5 and increased expression of aldehyde dehydrogenase encoded by ALD6 (Fig. 3, Table 1) in XUSEA-B during glucose fermentation. Meanwhile, the high availability of the cytosolic acetyl-CoA pool contributed substantially to the improved n-butanol production in XUSEA-B during glucose and glucose/xylose fermentation, thus demonstrating the advantage of employing xylose-utilizing $S$. cerevisiae for the production of acetyl-CoA-derived biofuels and biochemicals from lignocellulosic biomass.

In XUSEA-B, the oxidative pentose phosphate pathway, a major route for NADPH production (Stincone et al., 2015), appeared to be actively regulated via upregulation of ZWF1, SOL3, and GND1, possibly supporting the notion that improved NADPH availability improves metabolic flux through the NADPHdependent rate-limiting step in the n-butanol production pathway. Indeed, the n-butanol pathway in the glucose/xylose co-fermenting strain, containing an NADPH-preferring cytoEtr1 protein, was better supported by the sufficient NADPH pool present in the XUSEA-B strain.

In terms of glycerol-metabolism, expression of the glycerol-1-phosphatase gene (GPP1), which is important for glycerol biosynthesis, decreased by 2.9- to 3.7-fold (Fig. 3, Table 1), whereas transcription of the glycerol dehydrogenase gene ( $G C Y 1$ ), which participates in glycerol catabolism under micro-aerobic conditions, was increased by 3.98- to 14-fold. Similarly, suppressed glycerol synthesis combined with disruption of the ethanol-fermentation pathway reportedly stimulates n-butanol production (Lian et al., 2014; Swidah et al., 2015; Schadeweg and Boles, 2016b) by increasing the abundance of NADH, a driving force of the n-butanol pathway (Kim and Hahn, 2015; Schadeweg and Boles, 2016a). Furthermore, the upregulation of genes related to cofactor regeneration, such as $O Y E 3$,SER33, and YEF1, suggest that XUSEA-B maintained an optimal balance by varying redox cofactors, not only for efficient n-butanol production. Understanding the transcriptomic characteristics of XUSEA-B strain not only help to explain the improved butanol production during glucose/xylose fermentation, but also could offer engineering strategies to further improve the butanol titer in this minimally engineered strain of XUSEA-B. These engineering targets could also be used for rewiring biosynthetic routes to produce acetyl-CoA- derived chemicals in the context of biorefinery.

Previously, acetate supplementation increased n-butanol production in a native producer of Clostridium sp. by upregulating the expression levels of CoA-transferase genes, thereby increasing the availability of acetylCoA and butyryl-CoA, two main precursors of the n-butanol synthesis pathway (Chen and Blaschek, 1999). During lignocellulosic fermentation by $S$. cerevisiae, however, acetate is regarded as a major inhibitory compound that functions to limit the fermentation performance of $S$. cerevisiae, particularly during xylose fermentation (Ko et al., 2015). S. cerevisiae endogenously expresses ACS1 and ACS2, which encode acetylcoA synthetases to convert acetate into acetyl-CoA using ATP (van den Berg et al., 1996). Therefore, acetate supplementation could be expected to support additional carbon flux through the n-butanol production pathway. As expected, in the current study n-butanol production increased during glucose fermentation in the presence of acetate. During xylose fermentation, however, acetate supplementation did not positively affect n-butanol production, possibly due to insufficient detoxification and limited ATP generation by xylose (Casey et al., 2010). In fact, increased acetate concentration caused a decrease in the n-butanol titer during xylose fermentation (Fig. 4a). However, when an acetate-tolerant glucose/xylose co-fermenting strain, XUSAEA-B, was used the beneficial effects of acetate supplementation were recovered resulting in significantly improved n-butanol production (Fig. 5). Moreover, during lignocellulosic fermentation, XUSAEA-B produced 60.1 $\mathrm{mg} / \mathrm{L}$ of n-butanol, accounting for a $14 \%$ higher yield compared to that obtained during fermentation using synthetic media with the same concentrations of sugars $(52.8 \mathrm{mg} / \mathrm{L})$, possibly due to the positive effect of acetate in lignocellulosic hydrolysates. 


\section{Conclusion}

In this study, we investigated the production of the acetyl-coA-derived product, n-butanol, by a glucose/xylose co-fermenting $S$. cerevisiae strain. The transcription profiles of $S$. cerevisiaewith efficient xylose catabolism revealed a modified cellular network that better supported the generation of acetyl-CoA and cofactors required for n-butanol fermentation. Incorporating acetate catabolism further improved n-butanol production from lignocellulosic hydrolysates. Consequently, the results of this study show the potential of using a glucose/xylose co-fermenting strain and lignocellulosic biomass as more attractive production host and resource for biorefinery.

\section{Acknowledgments}

This research was supported by the Korea Institute of Science and Technology (KIST) Institutional Program [grant number 2E30170] and the National Research Foundation of Korea (NRF) funded by the Ministry of Science and ICT (Information \& Communication Technology) [grant number NRF-2020M1A2A2080847].

\section{Conflicts of interest statement}

The authors declare that the research was conducted in the absence of any commercial or financial relationships that could be construed as a potential conflict of interest.

\section{References}

Brat, D., \& Boles, E. (2013). Isobutanol production from D-xylose by recombinant Saccharomyces cerevisiae . FEMS yeast research , 13 (2), 241-244.doi: 10.1111/1567-1364.12028

Casey, E., Sedlak, M., Ho, N. W., \& Mosier, N. S. (2010). Effect of acetic acid and pH on the cofermentation of glucose and xylose to ethanol by a genetically engineered strain ofSaccharomyces cerevisiae. FEMS yeast research , 10 (4), 385-393.doi: 10.1111/j.1567-1364.2010.00623.x

Chen, C. K., \& Blaschek, H. P. (1999). Effect of acetate on molecular and physiological aspects of Clostridium beijerinckii NCIMB 8052 solvent production and strain degeneration. Applied and environmental microbiology , 65 (2), 499-505.doi: 10.1128/AEM.65.2.499-505.1999

Dellomonaco, C., Clomburg, J. M., Miller, E. N., \& Gonzalez, R. (2011). Engineered reversal of the $\beta$ oxidation cycle for the synthesis of fuels and chemicals. Nature , 476 (7360), 355-359.doi: 10.1038/nature10333

Ekas, H., Deaner, M., \& Alper, H. S. (2019). Recent advancements in fungal-derived fuel and chemical production and commercialization. Current opinion in biotechnology , 57 , 1-9. doi:10.1016/j.copbio.2018.08.014

González-Ramos, D., van den Broek, M., van Maris, A. J., Pronk, J. T., \& Daran, J. M. (2013). Genome-scale analyses of butanol tolerance in Saccharomyces cerevisiae reveal an essential role of protein degradation. Biotechnology for biofuels , 6 (1), 48. doi: 10.1186/1754-6834-6-48

Guo, W., Sheng, J., Zhao, H., \& Feng, X. (2016). Metabolic engineering of Saccharomyces cerevisiae to produce 1-hexadecanol from xylose. Microbial cell factories , 15 , 24. doi: 10.1186/s12934-016-0423-9

Hoang Nguyen Tran, P., Ko, J. K., Gong, G., Um, Y., \& Lee, S. M. (2020). Improved simultaneous cofermentation of glucose and xylose by Saccharomyces cerevisiae for efficient lignocellulosic biorefinery. Biotechnology for biofuels , 13 , 12.doi: 10.1186/s13068-019-1641-2

Hong, K. K., \& Nielsen, J. (2012). Metabolic engineering of Saccharomyces cerevisiae : a key cell factory platform for future biorefineries. Cellular and molecular life sciences : CMLS , 69 (16), 2671-2690.doi: 10.1007/s00018-012-0945-1

Kim, J., Hoang Nguyen Tran, P., \& Lee, S. M. (2020). Current Challenges and Opportunities in Non-native Chemical Production by Engineered Yeasts. Frontiers in bioengineering and biotechnology , 8, 594061. doi: 10.3389/fbioe.2020.594061 
Kim, S., \& Hahn, J. S. (2015). Efficient production of 2,3-butanediol in Saccharomyces cerevisiae by eliminating ethanol and glycerol production and redox rebalancing. Metabolic engineering , 31, 94-101.doi: 10.1016/j.ymben.2015.07.006

Knoshaug, E. P., \& Zhang, M. (2009). Butanol tolerance in a selection of microorganisms. Applied biochemistry and biotechnology , 153 (1-3), 13-20.doi: 10.1007/s12010-008-8460-4

Ko, J.K., Enkh, A.T., Gong, G.T., Um, Y.S., \& Lee, S.M. (2019). Improved bioconversion of lignocellulosic biomass by Saccharomyces cerevisiae engineered for tolerance to acetic acid. Global Change Biology , 12, 90-100.doi: $10.1111 / \mathrm{gcbb} .12656$

Ko, J. K., Jung, J. H., Altpeter, F., Kannan, B., Kim, H. E., Kim, K. H., Alper, H. S., Um, Y., \& Lee, S. M. (2018). Largely enhanced bioethanol production through the combined use of lignin-modified sugarcane and xylose fermenting yeast strain. Bioresource technology , 256 , 312-320.doi: 10.1016/j.biortech.2018.01.123

Ko, J. K., \& Lee, S. M. (2018). Advances in cellulosic conversion to fuels: engineering yeasts for cellulosic bioethanol and biodiesel production. Current opinion in biotechnology , 50, 72-80.doi: 10.1016/j.copbio.2017.11.007

Ko, J. K., Um, Y., Park, Y. C., Seo, J. H., \& Kim, K. H. (2015). Compounds inhibiting the bioconversion of hydrothermally pretreated lignocellulose. Applied microbiology and biotechnology , 99 (10), 4201-4212.doi: 10.1007/s00253-015-6595-0

Kwak, S., \& Jin, Y. S. (2017). Production of fuels and chemicals from xylose by engineeredSaccharomyces cerevisiae : a review and perspective. Microbial cell factories , 16 (1), 82.doi: 10.1186/s12934-017-0694-9

Li, X., Chen, Y., \& Nielsen, J. (2019). Harnessing xylose pathways for biofuels production. Current opinion in biotechnology , 57 , 56-65.doi: 10.1016/j.copbio.2019.01.006

Lian, J., Si, T., Nair, N. U., \& Zhao, H. (2014). Design and construction of acetyl-CoA overproducing Saccharomyces cerevisiaestrains. Metabolic engineering , 24 , 139-149. doi:10.1016/j.ymben.2014.05.010

Lian, J., \& Zhao, H. (2015). Reversal of the $\beta$-oxidation cycle in Saccharomyces cerevisiae for production of fuels and chemicals. ACS synthetic biology , 4 (3), 332-341. doi: 10.1021/sb500243c

Sakuragi, H., Morisaka, H., Kuroda, K., \& Ueda, M. (2015). Enhanced butanol production by eukaryotic Saccharomyces cerevisiaeengineered to contain an improved pathway. Bioscience, biotechnology, and biochemistry , 79 (2), 314-320.doi.org/10.1080/09168451.2014.972330

Schadeweg, V., \& Boles, E. (2016). Increasing n-butanol production with Saccharomyces cerevisiae by optimizing acetyl-CoA synthesis, NADH levels and trans-2-enoyl-CoA reductase expression. Biotechnology for biofuels , 9 , 257.doi: 10.1186/s13068-016-0673-0

Schadeweg, V., \& Boles, E. (2016). n-Butanol production in Saccharomyces cerevisiae is limited by the availability of coenzyme A and cytosolic acetyl-CoA. Biotechnology for biofuels , 9 , 44. doi: 10.1186/s13068016-0456-7

Stincone, A., Prigione, A., Cramer, T., Wamelink, M. M., Campbell, K., Cheung, E., Olin-Sandoval, V., Grüning, N. M., Krüger, A., Tauqeer Alam, M., Keller, M. A., Breitenbach, M., Brindle, K. M., Rabinowitz, J. D., \& Ralser, M. (2015). The return of metabolism: biochemistry and physiology of the pentose phosphate pathway. Biological reviews of the Cambridge Philosophical Society , 90 (3), 927-963. doi: 10.1111/brv.12140

Sun, L., \& Alper, H. S. (2020). Non-conventional hosts for the production of fuels and chemicals. Current opinion in chemical biology , 59 , 15-22. doi: 10.1016/j.cbpa.2020.03.004

Sun, L., Kwak, S., \& Jin, Y. S. (2019). Vitamin A Production by Engineered Saccharomyces cerevisiae from Xylose via Two-Phase in Situ Extraction. ACS synthetic biology , 8 (9), 2131-2140.doi: 10.1021/acssynbio.9b00217 
Swidah, R., Ogunlabi, O., Grant, C. M., \& Ashe, M. P. (2018). n-Butanol production in S. cerevisiae : co-ordinate use of endogenous and exogenous pathways. Applied microbiology and biotechnology , 102 (22), 9857-9866.doi: 10.1007/s00253-018-9305-x

Swidah, R., Wang, H., Reid, P. J., Ahmed, H. Z., Pisanelli, A. M., Persaud, K. C., Grant, C. M., \& Ashe, M. P. (2015). Butanol production in S. cerevisiae via a synthetic ABE pathway is enhanced by specific metabolic engineering and butanol resistance. Biotechnology for biofuels , 8 , 97.doi: 10.1186/s13068-015-0281-4

Tran Nguyen Hoang, P., Ko, J. K., Gong, G., Um, Y., \& Lee, S. M. (2018). Genomic and phenotypic characterization of a refactored xylose-utilizing Saccharomyces cerevisiae strain for lignocellulosic biofuel production. Biotechnology for biofuels , 11 , 268. doi: 10.1186/s13068-018-1269-7

van den Berg, M. A., de Jong-Gubbels, P., Kortland, C. J., van Dijken, J. P., Pronk, J. T., \& Steensma, H. Y. (1996). The two acetyl-coenzyme A synthetases of Saccharomyces cerevisiae differ with respect to kinetic properties and transcriptional regulation. The Journal of biological chemistry , 271 (46), 28953-28959.doi: $10.1074 / \mathrm{jbc} .271 .46 .28953$

Table 1 : Fold-changes in the expression levels of genes involved in metabolic pathways in XUSEA-B strain, versus the WT-B strain. G: glucose, GX: glucose and xylose

\begin{tabular}{|c|c|c|c|}
\hline Gene symbol & $\begin{array}{l}\text { Expression } \\
\text { fold-change }\end{array}$ & $\begin{array}{l}\text { Expression } \\
\text { fold-change }\end{array}$ & Function \\
\hline & G & GX & \\
\hline Glycolysis & Glycolysis & Glycolysis & Glycolysis \\
\hline TDH2 & 0.42 & 0.40 & $\begin{array}{l}\text { Glyceraldehyde-3- } \\
\text { phosphate } \\
\text { dehydrogenase }\end{array}$ \\
\hline$F B A 1$ & 0.49 & 0.50 & $\begin{array}{l}\text { Fructose } \\
\text { 1,6-bisphosphate } \\
\text { aldolase }\end{array}$ \\
\hline ENO2 & 0.43 & 0.48 & $\begin{array}{l}\text { Phosphopyruvate } \\
\text { hydratase }\end{array}$ \\
\hline $\begin{array}{l}\text { Pentose phosphate } \\
\text { pathway }\end{array}$ & $\begin{array}{l}\text { Pentose phosphate } \\
\text { pathway }\end{array}$ & $\begin{array}{l}\text { Pentose phosphate } \\
\text { pathway }\end{array}$ & $\begin{array}{l}\text { Pentose phosphate } \\
\text { pathway }\end{array}$ \\
\hline Oxidative branch & Oxidative branch & Oxidative branch & Oxidative branch \\
\hline$Z W F 1$ & 2.81 & 3.74 & $\begin{array}{l}\text { Glucose-6-phosphate } \\
\text { dehydrogenase }\end{array}$ \\
\hline SOL3 & 4.11 & 5.54 & $\begin{array}{l}6 \text { - } \\
\text { phosphogluconolactonase }\end{array}$ \\
\hline GND1 & 7.82 & 9.93 & $\begin{array}{l}\text { 6-phosphogluconate } \\
\text { dehydrogenase }\end{array}$ \\
\hline Non-oxidative branch & Non-oxidative branch & Non-oxidative branch & Non-oxidative branch \\
\hline$R P E 1$ & 100 & 95.1 & $\begin{array}{l}\text { D-ribulose-5-phosphate } \\
\text { 3-epimerase }\end{array}$ \\
\hline$X K S 1$ & 9.76 & 29.5 & Xylulokinase \\
\hline TAL1 & 3.15 & 3.96 & Transaldolase \\
\hline $\begin{array}{l}\text { Electron transport } \\
\text { change }\end{array}$ & $\begin{array}{l}\text { Electron transport } \\
\text { change }\end{array}$ & $\begin{array}{l}\text { Electron transport } \\
\text { change }\end{array}$ & $\begin{array}{l}\text { Electron transport } \\
\text { change }\end{array}$ \\
\hline$C Y C 1$ & 0.36 & 0.48 & $\begin{array}{l}\text { Cytochrome c, isoform } \\
1\end{array}$ \\
\hline$A A C 3$ & 2.24 & 2.2 & $\begin{array}{l}\text { Mitochondrial inner } \\
\text { membrane ADP /ATP } \\
\text { translocator }\end{array}$ \\
\hline
\end{tabular}




\begin{tabular}{|c|c|c|c|}
\hline Gene symbol & $\begin{array}{l}\text { Expression } \\
\text { fold-change }\end{array}$ & $\begin{array}{l}\text { Expression } \\
\text { fold-change }\end{array}$ & Function \\
\hline $\begin{array}{l}\text { TCA cycle and } \\
\text { glyoxylate cycle }\end{array}$ & $\begin{array}{l}\text { TCA cycle and } \\
\text { glyoxylate cycle }\end{array}$ & $\begin{array}{l}\text { TCA cycle and } \\
\text { glyoxylate cycle }\end{array}$ & $\begin{array}{l}\text { TCA cycle and } \\
\text { glyoxylate cycle }\end{array}$ \\
\hline CIT2 & 0.32 & 0.50 & Citrate synthase \\
\hline$A C O 1$ & 0.27 & 0.49 & Aconitase \\
\hline$P Y C 1$ & 0.29 & 0.42 & $\begin{array}{l}\text { Cytoplasmic pyruvate } \\
\text { carboxylase; } \\
\text { decarboxylates } \\
\text { pyruvate to } \\
\text { oxaloacetate }\end{array}$ \\
\hline PYC2 & 0.44 & 0.49 & $\begin{array}{l}\text { Cytoplasmic pyruvate } \\
\text { carboxylase; } \\
\text { decarboxylates } \\
\text { pyruvate to } \\
\text { oxaloacetate }\end{array}$ \\
\hline $\begin{array}{l}\text { Ethanol- } \\
\text { fermentation }\end{array}$ & $\begin{array}{l}\text { Ethanol- } \\
\text { fermentation }\end{array}$ & $\begin{array}{l}\text { Ethanol- } \\
\text { fermentation }\end{array}$ & $\begin{array}{l}\text { Ethanol- } \\
\text { fermentation }\end{array}$ \\
\hline $\mathrm{ADH}_{4}$ & 0.1 & 0.09 & $\begin{array}{l}\text { Alcohol dehydrogenase } \\
\text { isoenzyme IV }\end{array}$ \\
\hline$A D H 5$ & 0.39 & 0.43 & $\begin{array}{l}\text { Alcohol dehydrogenase } \\
\text { isoenzyme } \mathrm{V}\end{array}$ \\
\hline$P D C 6$ & 14.4 & 6.07 & $\begin{array}{l}\text { Pyruvate } \\
\text { decarboxylase; } \\
\text { decarboxylates } \\
\text { pyruvate to } \\
\text { acetaldehyde }\end{array}$ \\
\hline$A L D 6$ & 3.36 & 0.77 & $\begin{array}{l}\text { Cytosolic aldehyde } \\
\text { dehydrogenase }\end{array}$ \\
\hline Glycerol & Glycerol & Glycerol & Glycerol \\
\hline $\begin{array}{l}\text { biosynthesis and } \\
\text { degradation }\end{array}$ & $\begin{array}{l}\text { biosynthesis and } \\
\text { degradation }\end{array}$ & $\begin{array}{l}\text { biosynthesis and } \\
\text { degradation }\end{array}$ & $\begin{array}{l}\text { biosynthesis and } \\
\text { degradation }\end{array}$ \\
\hline$G P P 1$ & 0.34 & 0.27 & Glycerol-1-phosphatase \\
\hline$G C Y 1$ & 3.98 & 14 & Glycerol dehydrogenase \\
\hline Amino acid & Amino acid & Amino acid & Amino acid \\
\hline biosynthesis & biosynthesis & biosynthesis & biosynthesis \\
\hline ILV3 & 0.28 & 0.39 & $\begin{array}{l}\text { Dihydroxy-acid } \\
\text { dehydratase }\end{array}$ \\
\hline$E E B 1$ & 0.14 & 0.28 & $\begin{array}{l}\text { Medium-chain fatty } \\
\text { acid ethyl ester } \\
\text { synthase/esterase }\end{array}$ \\
\hline$L E U 1$ & 0.26 & 0.43 & $\begin{array}{l}\text { Isopropylmalate } \\
\text { isomerase }\end{array}$ \\
\hline LEU2 & 0.29 & 0.30 & $\begin{array}{l}\text { Beta-isopropylmalate } \\
\text { dehydrogenase }\end{array}$ \\
\hline LEU9 & 0.36 & 0.26 & $\begin{array}{l}\text { Alpha-isopropylmalate } \\
\text { synthase I }\end{array}$ \\
\hline$T R P \mathscr{2}$ & 0.46 & 0.39 & Anthranilate synthase \\
\hline Other functions & Other functions & Other functions & Other functions \\
\hline
\end{tabular}




\begin{tabular}{llll}
\hline Gene symbol & $\begin{array}{l}\text { Expression } \\
\text { fold-change }\end{array}$ & $\begin{array}{l}\text { Expression } \\
\text { fold-change }\end{array}$ & Function \\
\hline OYE3 & 7.16 & 12.21 & $\begin{array}{l}\text { NADPH } \\
\text { oxidoreductase } \\
\text { containing a flavin } \\
\text { mononucleotide } \\
\text { 3-phosphoglycerate } \\
\text { dehydrogenase }\end{array}$ \\
SER33 & 3.88 & 3.44 & $\begin{array}{l}\text { ATP-NADH kinase; } \\
\text { phosphorylates both } \\
\text { NEF1 }\end{array}$ \\
3.69 & 7.64 & $\begin{array}{l}\text { NAD and NADH } \\
\text { Cytosolic acetyl-CoA } \\
\text { C-acetyltransferase }\end{array}$ \\
\hline
\end{tabular}

Table 2. Strains used in this study

\begin{tabular}{|c|c|c|}
\hline Strain & \multicolumn{2}{|c|}{ Description } \\
\hline \multicolumn{3}{|l|}{ E. coli } \\
\hline \multirow{2}{*}{$\begin{array}{l}\mathrm{DH} 10 \beta \\
\text { S. cerevisiae }\end{array}$} & \multicolumn{2}{|r|}{$\mathrm{F}^{-} \mu \varsigma \rho A \Delta\left(\mu \rho \rho-\eta \sigma \delta P M \Sigma-\mu \varsigma \rho B^{\circ}\right) \varphi 80 \lambda a \varsigma Z \Delta M 15 \Delta \lambda a_{\varsigma} \Xi 74 \rho \epsilon \varsigma A 1 \epsilon \nu \delta A 1$ a $\rho a \Delta 139$} \\
\hline & & \\
\hline BY4741 & \multicolumn{2}{|c|}{ 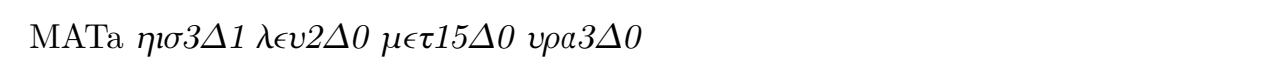 } \\
\hline BYB & \multicolumn{2}{|c|}{ BY4741 harboring P423-Hbd-Crt, P425-Erg10-cyto Etr1, and P426-BdhB-EutE } \\
\hline XUSEA & \multicolumn{2}{|c|}{ BY4741 $x y l A^{*} 3$ TAL1 XKS1 RPE1 $\triangle y \rho \in 3 \Delta \pi \eta \circ 13 \Delta a \sigma \varsigma 1$ evolved strain } \\
\hline $\mathrm{XA}-1$ & \multicolumn{2}{|c|}{ XUSEA harboring $\mathrm{P} 423$ - $_{\text {cyto }} K r_{\text {- }}{ }_{\text {cyto }} H t d, \mathrm{P} 425-E r g 10$ - $_{\text {cyto }} E \operatorname{tr} 1$, and $\mathrm{P} 426-A d h E 2-E$} \\
\hline $\mathrm{XA}-2$ & \multicolumn{2}{|c|}{ XUSEA harboring $\mathrm{P} 423$ - $_{\text {cyto }} K r_{\text {-cyto }} H t d, \mathrm{P} 425-E r g 10$ - $_{\text {cyto }} E \operatorname{tr} 1$, and P426-AdhE2 } \\
\hline $\mathrm{XA}-3$ & \multicolumn{2}{|c|}{ 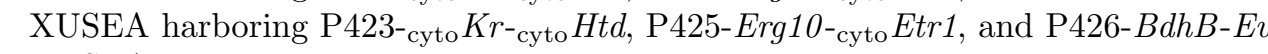 } \\
\hline $\mathrm{XA}-4$ & \multicolumn{2}{|c|}{ XUSEA harboring P423- cyto $K r_{- \text {cyto }} H t d, \mathrm{P} 425-E r g 10$ - $_{\text {cyto }}$ Etr 1 , and $\mathrm{P} 426-B d h B$} \\
\hline XA -5 & \multicolumn{2}{|c|}{ XUSEA harboring P423-Hbd-Crt, P425-Erg10-cyto Etr1, and P426-AdhE2-EutE } \\
\hline XA - 6 & \multicolumn{2}{|c|}{ XUSEA harboring P423-Hbd-Crt, P425-Erg10-cyto Etr1, and P426-AdhE2 } \\
\hline XA-7 (XUSEA-B) & \multicolumn{2}{|c|}{ XUSEA harboring P423-Hbd-Crt, P425-Erg10-cyto Etr1, and P426-BdhB-EutE } \\
\hline $\mathrm{XA}-8$ & \multicolumn{2}{|c|}{ XUSEA harboring P423-Hbd-Crt, $\mathrm{P} 425-E r g 10$-cyto $E t r 1$, and $\mathrm{P} 426-B d h B^{-}$} \\
\hline XA -Ter & \multicolumn{2}{|c|}{ XUSEA harboring P423-Hbd-Crt-Ter and P426-BdhB-EutE-Erg10 } \\
\hline $\mathrm{XA}-_{\text {cyto }}$ Etr1 & \multicolumn{2}{|c|}{ XUSEA harboring P423-Hbd-Crt-cyto Etr1 and P426-BdhB-EutE-Erg10 } \\
\hline \multicolumn{3}{|c|}{ Table 3. Plasmids used in this study } \\
\hline \multicolumn{2}{|l|}{ Plasmid } & Characteristics \\
\hline$\overline{\mathrm{P} 423-_{\text {cyto }} K r_{-\mathrm{cy}}}$ & & GPDp-cyto $_{-} K r$-PRM9t and TEFp- ${ }_{\text {cyto }} H t d$-CPS1t \\
\hline $\mathrm{P} 423-\mathrm{Hbd}-\mathrm{Crt}$ & & GPDp-Hbd-PRM9t and TEFp-Crt-CPS1t \\
\hline $\mathrm{P} 423-\mathrm{Hbd}-\mathrm{Crt}-$ & & GPDp-Hbd-PRM9t, TEFp-Crt-CPS1t, and HXT7p-Ter-TPI7t \\
\hline $\mathrm{P} 423-H b d-C r t-$ & $\operatorname{Ter}{ }^{\mathrm{G} 155 \mathrm{C}}$ & GPDp-Hbd-PRM9t, TEFp-Crt-CPS1t, and HXT7p-Ter G155C-TPI7t \\
\hline $\mathrm{P} 423-\mathrm{Hbd}-\mathrm{Crt}_{-}$ & cyto Etr1 & GPDp-Hbd-PRM9t, TEFp-Crt-CPS1t, and HXT7p-cyto Etr1-TPI7t \\
\hline $\mathrm{P} 425-E r g 10-$ cyt & Etr1 & TEFp-Erg10-CYC1t and HXT7p-cyto Etr1-TPI7t \\
\hline $\mathrm{P} 426-A d h E_{2}$ & & CYC1p-AdhE2-SPG5t \\
\hline $\mathrm{P} 426-A d h E 2-E$ & & CYC1p-AdhE2-SPG5t and PGK1p-EutE-CYC1t \\
\hline $\mathrm{P} 426-B d h B$ & & CYC1p- $B d h B-$ SPG5t \\
\hline $\mathrm{P} 426-B d h B-E u$ & & CYC1p- $B d h B$-SPG5t and PGK1p-EutE-CYC1t \\
\hline $\mathrm{P} 426-B d h B-E u$ & $t E-\operatorname{Erg} 10$ & CYC1p-BdhB-SPG5t, PGK1p-EutE-CYC1t, and TEFp-Erg10-CYC1t \\
\hline
\end{tabular}




\section{Figure legend :}

Fig. 1. n-butanol production in the glucose/xylose co-fermenting S. cerevisiae strain, XUSEA, expressing various gene sets used for n-butanol biosynthesis. The genes were sourced fromS. cerevisiae $(S c)$, Y. lipolytica $(Y l)$,E. coli $(E c)$, and $C$. acetobutyricum $(C a)$. Low cell density fermentations (initial OD of 0.2 ) were conducted with $20 \mathrm{~g} / \mathrm{L}$ xylose as the sole carbon source. (a) n-butanol pathway showing the genes used in this study. (b) Six combinations of genes used to construct various n-butanol production pathways. The error bars represent the standard deviations obtained using biological triplicates. *: below the detection limit of $2.5 \mathrm{mg} / \mathrm{L}$

Fig. 2. Comparison of n-butanol production between the WT-B and XUSEA-B strains during glucose and/or xylose fermentation. Fermentation was conducted with an initial OD of 0.2. (a) n-butanol titer after $72 \mathrm{~h}$ of fermentation. (b) Glucose (circles)/xylose (triangles) consumption and ethanol production (squares) during co-fermentation. The error bars represent the standard deviations obtained using biological triplicates.

Fig. 3. Improved central carbon flux toward the butanol pathway in XUSEA-B strain. Arrows indicate the changes of gene transcription level in XUSEA-B compared to those in WT-B strain. The cells were grown on glucose medium (20 g/L glucose) or a mixed-sugar medium $(20 \mathrm{~g} / \mathrm{L}$ glucose and $20 \mathrm{~g} / \mathrm{L}$ xylose $)$ under micro-aerobic conditions. Sampling was conducted during the exponential phase of cell growth. The gene symbols were referenced from the Saccharomyces Genome Database.

Fig. 4. Effect of acetate supplementation on n-butanol production by XUSEA-B strains during glucose and/or xylose fermentation. Fermentation was conducted with an initial OD of 0.2 and various concentrations of acetate $(0,1$, or $2 \mathrm{~g} / \mathrm{L})$. (a) n-butanol production during glucose (pattern, blue) or xylose (solid, yellow) fermentation. (b) n-butanol production during glucose and xylose co-fermentation with (yellow) and without (white) acetate supplementation $(0 \mathrm{~g} / \mathrm{L}, 0.2 \mathrm{~g} / \mathrm{L}, 0.5 \mathrm{~g} / \mathrm{L}$, or $1 \mathrm{~g} / \mathrm{L})$. The error bars represent standard deviations obtained using biological triplicates.

Fig. 5. Comparison of n-butanol production by the XUSEA-B strain and acetate-tolerant strains of XUSAEA-B during glucose/xylose co-fermentation. Fermentation was conducted using an initial OD of 0.2 with $0 \mathrm{~g} / \mathrm{L}$ (white) and $1 \mathrm{~g} / \mathrm{L}$ (pattern, blue) of acetate supplementation at $\mathrm{pH}$ 5.0. n-butanol production at $\mathrm{pH} 6.5$ was also evaluated with $1 \mathrm{~g} / \mathrm{L}$ of acetate supplementation (black). The error bars represent standard deviations obtained using biological triplicates.

Fig. 6. n-butanol fermentation by the XUSAEA-B strain using lignocellulosic hydrolysates. Fermentation was conducted withMiscanthus hydrolysates pretreated with diluted acids. Fermentation with synthetic medium composed of the same concentration of sugars $(30 \mathrm{~g} / \mathrm{L}$ of glucose and $15 \mathrm{~g} / \mathrm{L}$ of xylose) was also conducted under the same conditions. The initial OD and $\mathrm{pH}$ were 15 and 6.5, respectively. (a) Fermentation performance using lignocellulosic hydrolysates (yellow). (b) Fermentation performance using synthetic hydrolysates (blue). (c) n-butanol production during fermentation using lignocellulosic hydrolysates (solid line, diamonds) and synthetic hydrolysate (dash line, diamonds). Ethanol production (squares) and glucose (circles) and xylose (triangles) consumption were measured during fermentation. The error bars represent standard deviations obtained using biological triplicates. 
a

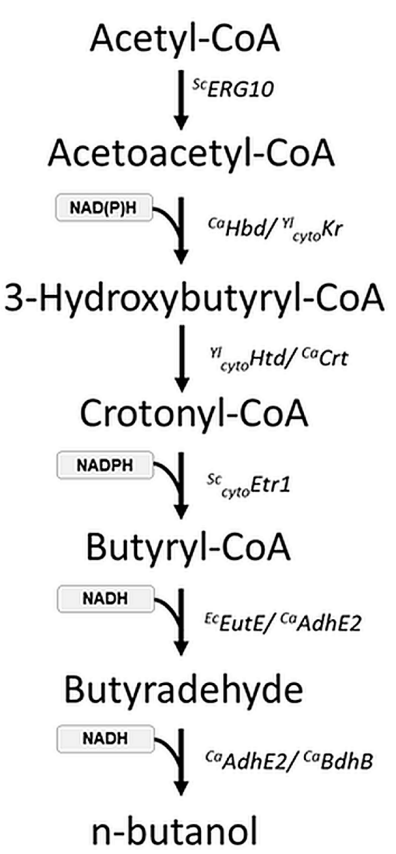

b

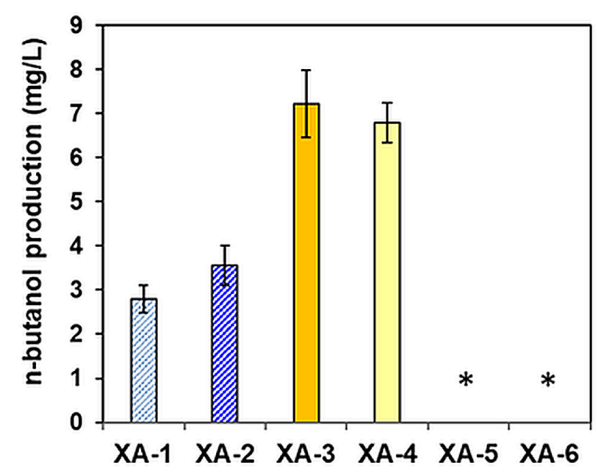

\begin{tabular}{|c|c|c|c|c|c|}
\hline${ }^{S_{C}} E R G 10$ & + & + & + & + & + \\
\hline${ }^{C a} \mathrm{Hbd}$ & - & - & + & + & - \\
\hline$r_{\text {cyto }} K r$ & + & + & - & - & + \\
\hline $\mathrm{YI}_{\text {cyto }} \mathrm{Htd}$ & + & + & - & - & + \\
\hline${ }^{c a} \mathrm{Crt}$ & - & - & + & + & - \\
\hline Sc ${ }_{\text {cyto }}$ Etr1 & + & + & + & + & + \\
\hline${ }^{E c}$ EUtE & + & + & + & + & - \\
\hline${ }^{C a} A d h E 2$ & + & - & + & - & + \\
\hline${ }^{C a} B d h B$ & - & + & - & + & - \\
\hline
\end{tabular}

b

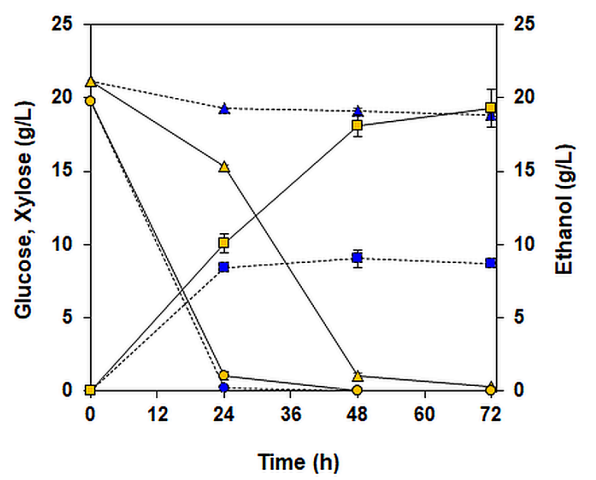



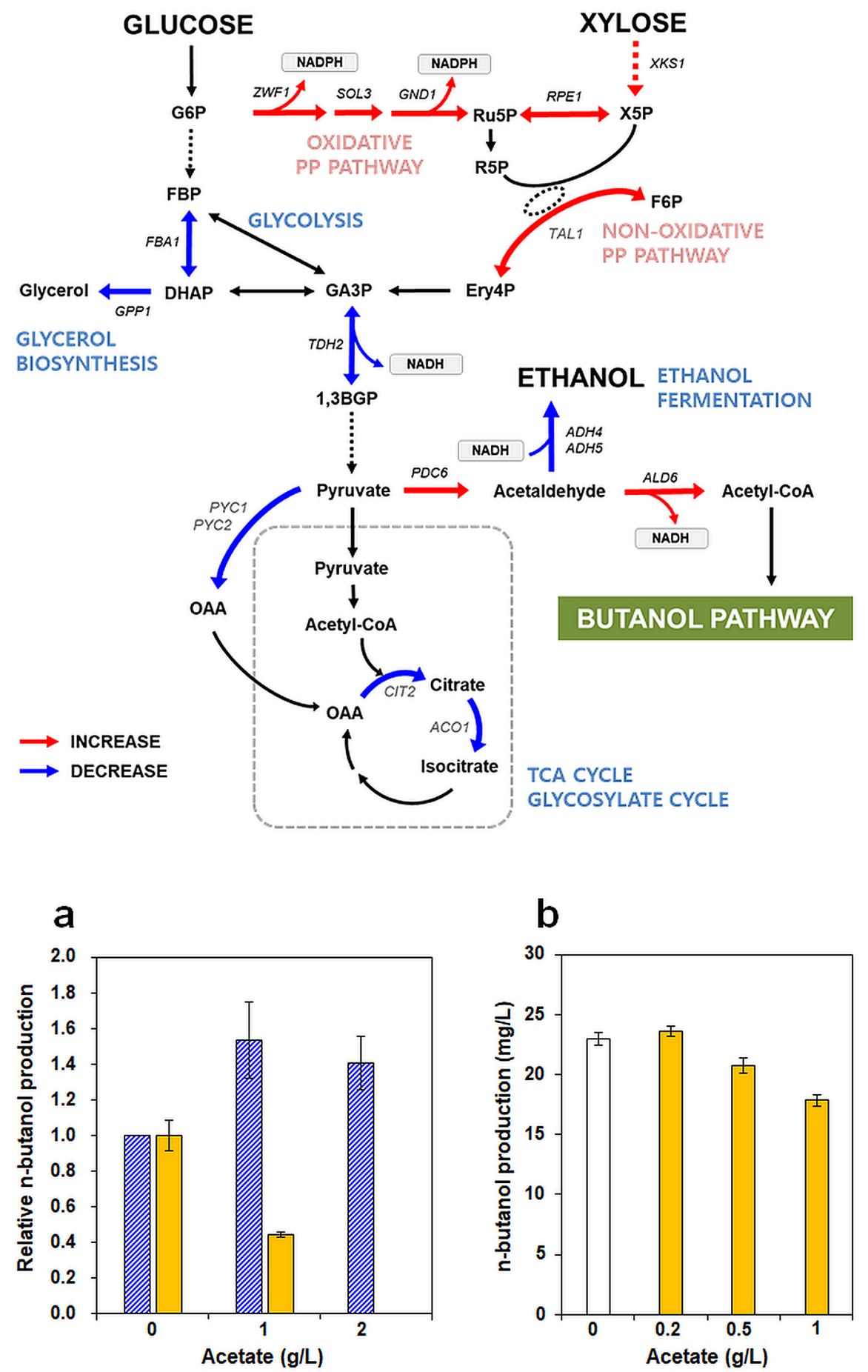

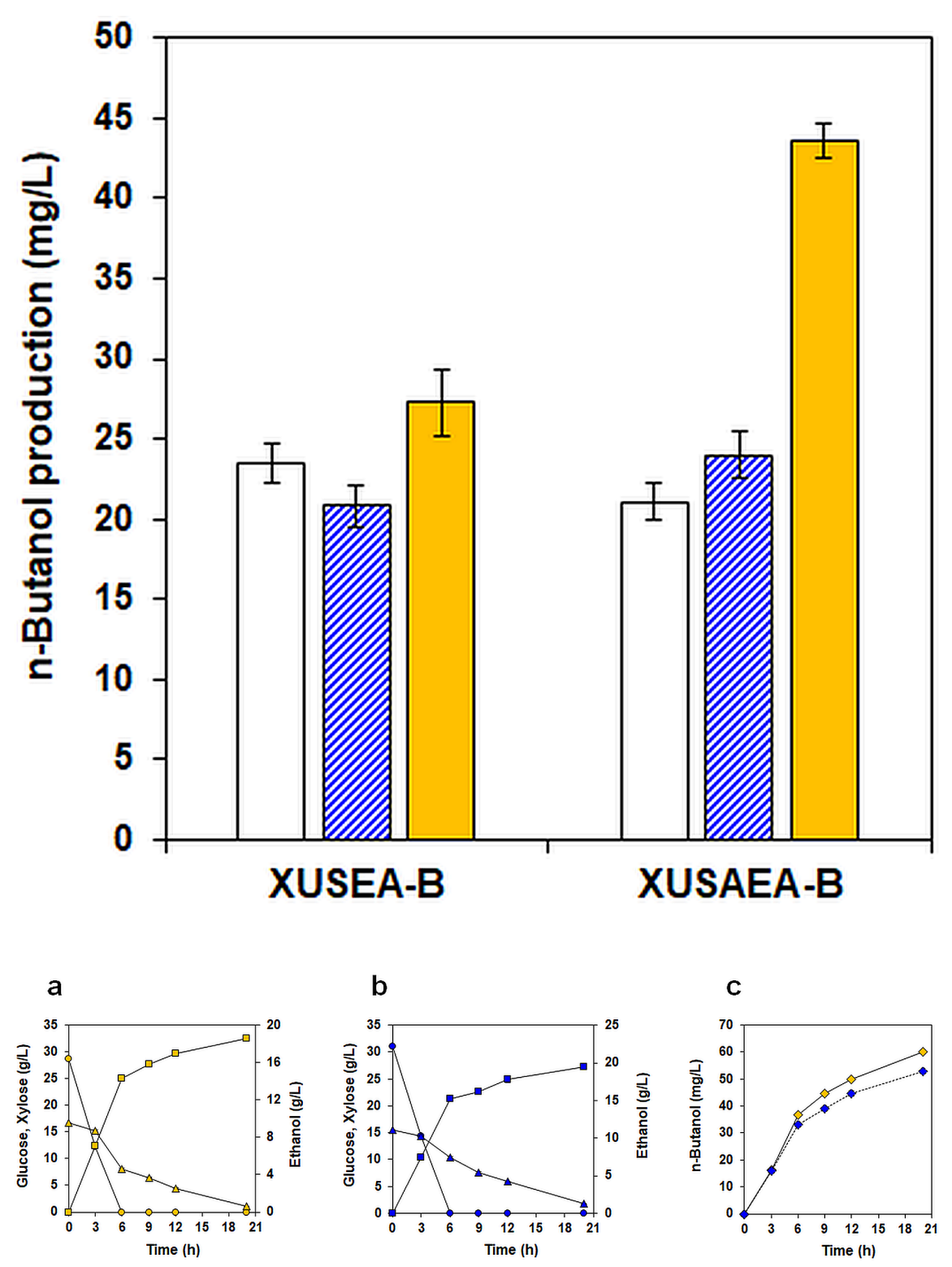


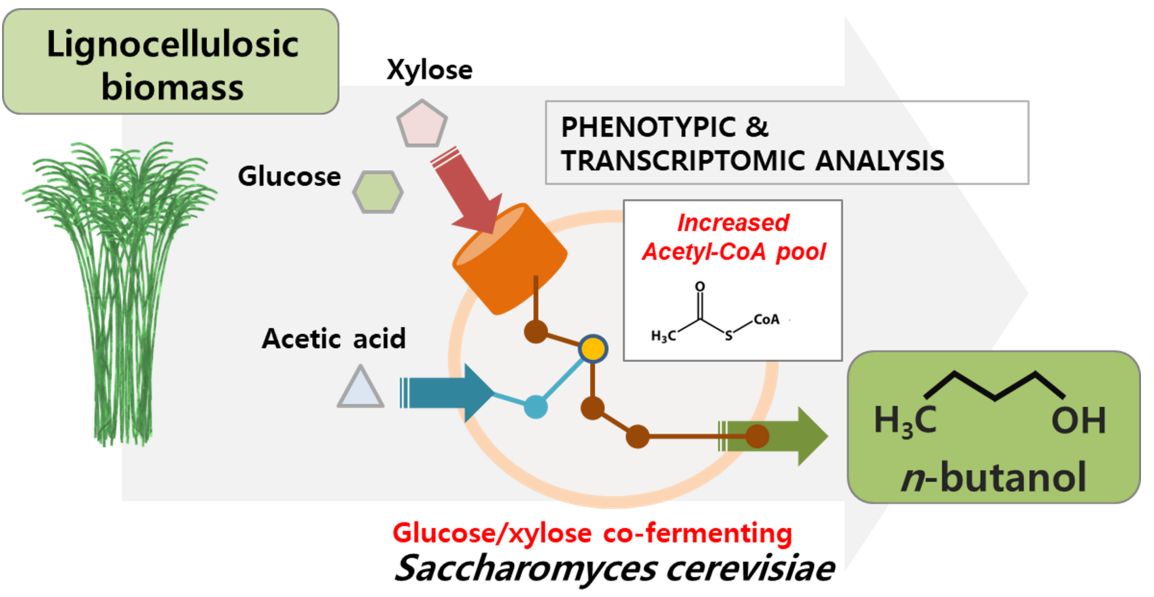

